Open Access

\title{
A Thief Caught in the Act
}

- Free Floating Venous Thrombus in the Right Heart Associated With Pulmonary Embolism -

\author{
Seung-Pyo Lee, MD, Hyung-Kwan Kim, MD, Song-Yi Kim, MD, Il-Young Oh, MD, \\ Hyun-Jai Cho, MD, Yong-Jin Kim, MD and Dae-Won Sohn, MD \\ Department of Internal Medicine and Cardiovascular Center, Seoul National University College of Medicine, Seoul, Korea
}

A 65-year-old, previous healthy woman was referred for evaluation of exertional dyspnea that had developed three days prior. Radical hysterectomy had been done for cervical cancer, followed by reoperation for wound dehiscence 12 days before referral. Lower extremity edema was observed solely on the right side. Transthoracic echocardiography revealed serpentine, free-floating thrombus, starting from the inferior vena cava and extending to the right ventricle (RV) at diastole and the dilated RV showed systolic dysfunction (Fig. 1A, B and C). Computed tomography (CT) showed near total obstruction of pulmonary arteries and deep vein thrombosis of the right femoral vein (Fig. 1D and E). Administration of intravenous heparin with close monitoring was selected as the initial treatment, rather than thrombolysis or surgical embolectomy. This was due to the patient's stable vital signs, and most importantly, a history of repeated surgical operation and serious wound dehiscence with ongoing infective intraabdominal lymphocele. The thrombus disappeared 4 days later, though pulmonary hypertension remained (Fig. $1 F$ and $G$ ). Oral anticoagulation treatment was started and the patient was markedly relieved of dyspnea 14 days later, with significant improvement of RV function (Fig. 1H) and complete resolution of pulmonary thrombus as assessed 4 months later by CT (Fig. 1I).

Although the prognosis is more fatal for patients with pulmonary embolism that is associated with right heart thrombus, ${ }^{1)}$ the efficacy of different treatments, including the use of anticoagulants, thrombolytic therapy, ${ }^{2)}$ or surgical embolectomy, ${ }^{3)}$ remains controversial. ${ }^{344}$ In this case, given the patient's stable vital signs and past medical history, radical thrombolysis or thrombectomy was not performed, and fortunately she had an uncomplicated recovery.

\section{REFERENCES}

1) Torbicki A, Galie N, Covezzoli A, et al. Right heart thrombi in pulmonary embolism: results from the International Cooperative Pulmonary Embolism Registry. J Am Coll Cardiol 2003;41:2245-51.

2) John SH, Jung GM, Choi HJ, et al. A case of huge right atrial thrombi treated with thrombolytic agent. Korean Circ J 2004;34:328-32.

3) Rose PS, Punjabi NM, Pearse DB. Treatment of right heart thromboemboli. Chest 2002;121:806-14.

4) Kinney EL, Wright RJ. Efficacy of treatment of patients with echocardiographically detected right-sided heart thrombi: a meta-analysis. Am Heart J 1989;118:569-73.

Received: April 21, 2010 / Revision Received: May 10, 2010 / Accepted: May 31, 2010

Correspondence: Hyung-Kwan Kim, MD, Department of Internal Medicine and Cardiovascular Center, Seoul National University College of Medicine, 101 Daehak-ro, Jongno-gu, Seoul 110-744, Korea

Tel: 82-2-2072-0243, Fax: 82-2-2072-3757, E-mail: cardiman@medimail.co.kr

- The authors have no financial conflicts of interest.

@ This is an Open Access article distributed under the terms of the Creative Commons Attribution Non-Commercial License (http://creativecommons.org/licenses/by-nc/3.0) which permits unrestricted non-commercial use, distribution, and reproduction in any medium, provided the original work is properly cited. 

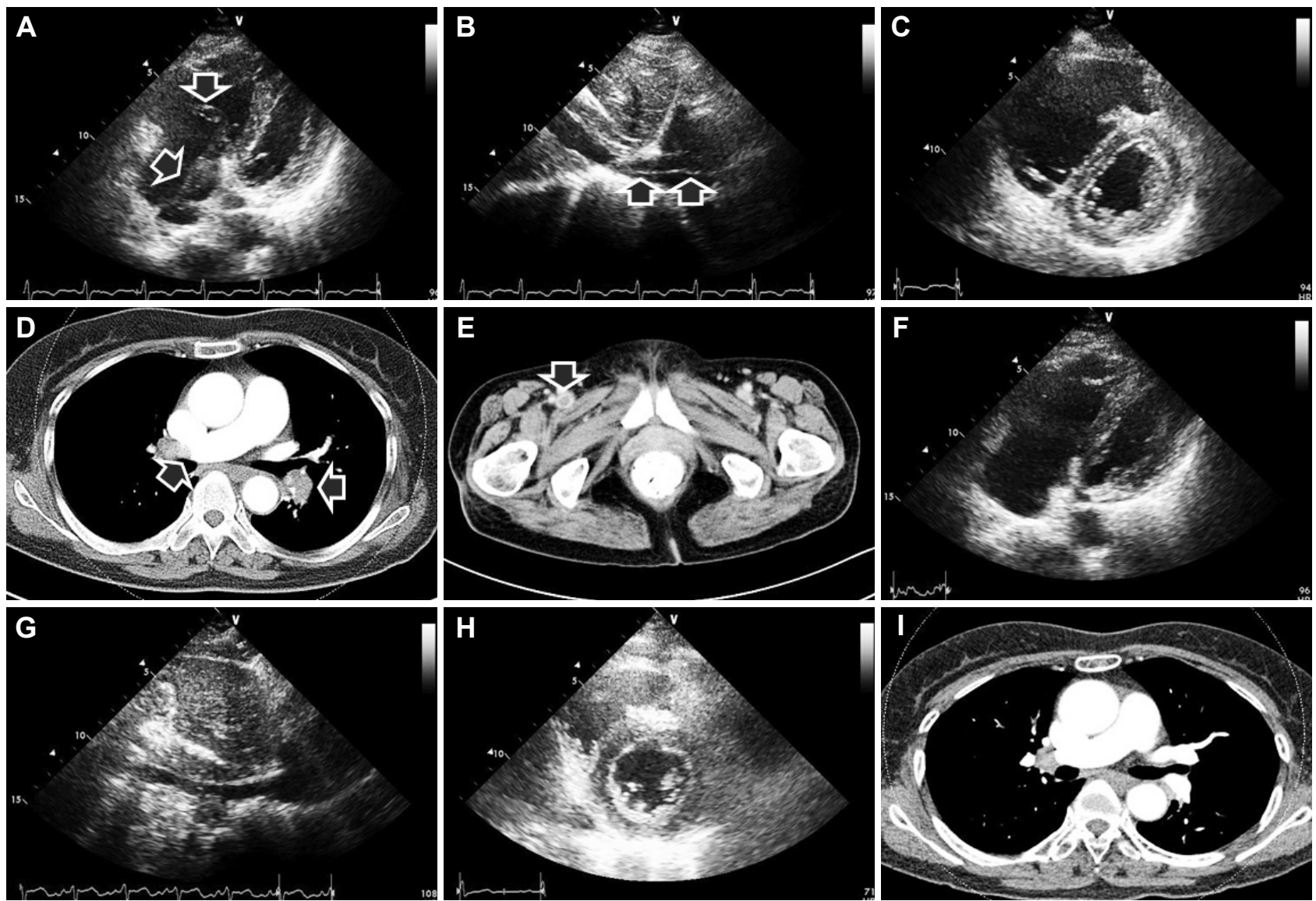

Fig. 1. Representative echocardiography and computed tomography images at initial presentation (A-E) and at follow-up (F-I). A and B: a large, serpentine, free-floating thrombus (arrows) was noted at the dilated right artium and ventricle (A) and also, inferior vena cava (B). C: the right ventricle was dilated and compressed the left ventricle in to a typical D-shape. D, E and F: thrombus was demonstrated clearly at both pulmonary arteries (arrows) (D) and also at the right femoral vein (arrows) (E) on computed tomography, confirming the diagnosis of pulmonary thromboembolism with concomitant deep vein thrombosis. F and $\mathrm{G}$ : the free-floating thrombus had disappeared from the right heart (F) and also, the inferior vena cava (G) 4 days after continuous intravenous heparin. $\mathrm{H}$ : the right and left ventricle had recovered into a normal configuration 14 days after oral anticoagulation. I: thrombus at pulmonary arteries had disappeared after 4 months of oral anticoagulation. 